\title{
Importance of 6-mercaptopurine dose in lymphoblastic leukaemia
}

Department of Haematology, The Children's Hospital, Western Bank, Sheffield S10 2 TH J P Hale

J S Lilleyman

Correspondence to: Dr Lilleyman.

Accepted 7 November 1990

\author{
J P Hale, J S Lilleyman
}

\begin{abstract}
To explore the possibility that higher total dosage of 'maintenance' treatment may have contributed to the recent improvement in outlook of children in the United Kingdom with lymphoblastic leukaemia, details of the amount of 6-mercaptopurine prescribed during the first two years of treatment were studied in an unselected cohort of children diagnosed between 1973 and 1987. Eighty five patients were studied, 30 diagnosed before and 55 after 1980. The group diagnosed after 1980 showed an $18 \%$ improvement in relapse free survival at five years. Their median total dose of 6-mercaptopurine had increased by $22 \%$, whereas according to the protocol it should have risen by an average of only $9 \%$. After 1980 boys were prescribed significantly more 6-mercaptopurine than girls, and had fewer dose reductions because of myelosuppression.
\end{abstract}

These findings support the clinical impression that after 1980 an important therapeutic difference resulting from the new United Kingdom acute lymphoblastic leukaemia protocols was an increase in the amount of 6 . mercaptopurine that children actually received as a result of changes in prescribing guidelines rather than dose. They also provide further evidence that boys tolerate 6-mercaptopurine better than girls, which may be related to the still unexplained difference in prognosis between the sexes.

During the last decade there has been a striking improvement in long term survival for children with lymphoblastic leukaemia in the United Kingdom. ${ }^{1}$ Before 1980 treatment protocols were somewhat vague about what modifications in dose were permitted during the 'maintenance' phase of treatment, with the result that many clinicians veered away from doses that caused myelosuppression to minimise morbidity. With the implementation of the United Kingdom acute lymphoblastic leukaemia (UKALL) VIII protocol in 1980, which required a much greater degree of compliance by doctors to a strict regimen of permitted modifications in doses, two notable differences gradually became apparent. Firstly, there was an increased incidence of morbidity and mortality as a result of the treatment and secondly, despite this, there was a $20 \%$ improvement in long term survival. ${ }^{1}$

The two antileukaemic drugs that were most affected by the changes in prescribing practice were 6-mercaptopurine and methotrexate, both of which were used during the 'maintenance' phase of treatment. Of these 6-mercaptopurine was likely to be the most important as the dose of methotrexate was relatively small.

It is important to understand that the main differences between protocols before and after 1980 are related to adjustments in dose rather than 'target' protocol doses. Our hypothesis was, therefore, that children treated for acute lymphoblastic leukaemia since 1980 had been prescribed more 6-mercaptopurine by having the dose reduced less frequently and that this could be an important factor in their better prognosis. We also suspected that, based on preliminary work on the pharmacokinetics of 6 mercaptopurine, ${ }^{2}$ there may be a difference between the sexes in tolerance of 6mercaptopurine, and it was to explore these two suppositions that the present study was undertaken.

\section{Patients and methods}

PATIENTS

Children with acute lymphoblastic leukaemia who were attending the Children's Hospital, Sheffield and who had completed two years continuing chemotherapy in their first remission were studied. The patients were divided into two groups, those diagnosed before 1980 (December 1973 to February 1980) and those diagnosed after 1980 (March 1980 to April 1990). In the group diagnosed before 1980 consecutive cases who received chemotherapy according to the Medical Research Council trial protocols UKALL III (arms 'ordinary' A, B, C, and $D$ and 'ordinary modified' $A$ and $E)^{3}$ and UKALL V (arms 'continuous' and 'gaps') ${ }^{4}$ were studied. In the group diagnosed after 1980 children receiving chemotherapy according to protocols UKALL VIII (arms A and B) ${ }^{1}$ and UKALL X (arm A) were studied. Only arm A of UKALL $X$ was used because the effect on the outcome of treatment of the additional blocks of intensification treatment in arms B, C, and $D$ of the trial was not known. Only children with no mediastinal mass and diagnostic white cell counts of less than $20 \times 10^{9} / 1$ were eligible for UKALL III and V, so when comparisons were made between patients diagnosed before and after 1980, children with T-cell acute lymphoblastic leukaemia and white cell counts of more than $20 \times 10^{9} / 1$ at diagnosis were excluded from the group diagnosed after 1980 . When the group diagnosed after 1980 was studied on its own, children with all immunological types of acute lymphoblastic leukaemia (except B-cell) and all diagnostic white cell counts were included. 
Medical Research Council trials in childhood acute lymphoblastic leukaemia

\begin{tabular}{|c|c|c|}
\hline Trial & 'Maintenance' regimen of 6-mercaptopurine & $\begin{array}{l}\text { Maximum cumulative } \\
\text { dose of 6-mercaptopurine } \\
\left(m g / m^{2}\right) \text { at eight } \\
\text { 'maintenance' cycles }\end{array}$ \\
\hline UKALL III (ordinary A,B,C,D) & $75 \mathrm{mg} / \mathrm{m}^{2} /$ day continuously from week 4 & 52500 \\
\hline $\begin{array}{l}\text { UKALL III: } \\
\text { Modified A } \\
\text { Modified E }\end{array}$ & $\begin{array}{l}25 \mathrm{mg} / \mathrm{m}^{2} / \text { day, weeks } 4-9 \\
75 \mathrm{mg} / \mathrm{m}^{2} / \text { day from week } 13 \\
25 \mathrm{mg} / \mathrm{m}^{2} / \text { day, weeks } 4-9 \\
75 \mathrm{mg} / \mathrm{m}^{2} / \text { day from week } 13 \text { with one week gap every four weeks }\end{array}$ & $\begin{array}{l}51450 \\
34650\end{array}$ \\
\hline $\begin{array}{l}\text { UKALL V: } \\
\text { Continuous } \\
\text { Gaps }\end{array}$ & $\begin{array}{l}25 \mathrm{mg} / \mathrm{m}^{2} / \text { day, weeks } 5-8 \\
50-70 \mathrm{mg} / \mathrm{m}^{2} / \text { day from week } 12 \\
25 \mathrm{mg} / \mathrm{m}^{2} / \text { day, weeks } 5-8 \\
70-100 \mathrm{mg} / \mathrm{m}^{2} / \text { day from week } 12 \text { with one week gap every } \\
\text { three weeks }\end{array}$ & $\begin{array}{l}47915 \\
45675\end{array}$ \\
\hline UKALL VIII $(A+B)$ & $75 \mathrm{mg} / \mathrm{m}^{2} /$ day from week 5 & 52500 \\
\hline UKALL XA & $75 \mathrm{mg} / \mathrm{m}^{2} /$ day from week 6 & 51975 \\
\hline
\end{tabular}

Children were studied from the time that they started receiving 6-mercaptopurine during the phase of treatment directed towards central nervous system prophylaxis and throughout eight subsequent cycles of 'maintenance' chemotherapy. All the protocols are described in detail elsewhere, ${ }^{13} 4$ and the differences in 6-mercaptopurine regimens among them are shown in the table. All contained an induction phase of four to five weeks when they received weekly vincristine intravenously $\left(1.5 \mathrm{mg} / \mathrm{m}^{2}\right)$, daily oral prednisolone $\left(40 \mathrm{mg} / \mathrm{m}^{2}\right)$, and asparaginase (crisantaspase) intramuscularly, the doses and times varying depending on the protocol. UKALL VIII $A$ and $X$ also included two intravenous doses of daunorubicin $\left(45 \mathrm{mg} / \mathrm{m}^{2}\right)$. Induction was followed by a central nervous system prophylaxis phase of two to three weeks during which cranial radiotherapy was given (24 Gy in UKALL III and V, and $18 \mathrm{~Gy}$ in UKALL VIII and X). This was accompanied by weekly intrathecal methotrexate, and 6mercaptopurine was started during this phase. 6-Mercaptopurine was given as a single daily oral dose, the dose being dependent on the protocol. Treatment was continued either continuously (UKALL III 'ordinary' and modified A, VIII, X and V 'continuous') or with a one week gap every three (UKALL III modified E) or four weeks (UKALL V 'gaps'), together with weekly methotrexate orally $\left(20 \mathrm{mg} / \mathrm{m}^{2}\right)$ and monthly vincristine intravenously $\left(1.5 \mathrm{mg} / \mathrm{m}^{2}\right)$ with five days of oral prednisolone $\left(40 \mathrm{mg} / \mathrm{m}^{2}\right)$. 'Maintenance' treatment was given for two to three years depending on the protocol.

In all the protocols the dose of 6mercaptopurine was reduced if myelosuppression occurred. In UKALL III and V the aim was to keep the total white cell count at 2-3X $10^{9} / 1$, but instructions were not specific and modification of the dose was largely at the clinicians' discretion.

In UKALL VIII and X much stricter criteria for modifying the doses of 6-mercaptopurine and methotrexate were introduced; the drugs were reduced to $50 \%$ or $0 \%$ of the target dose if the neutrophil count fell to less than 1 or less than $0.5 \times 10^{9} / 1$. They were restarted when the counts rose above the threshold and built up to the target dose again over a period of three weeks.
In the latter part of UKALL VIII and throughout UKALL $\mathrm{X}$ oral co-trimoxazole was given during 'maintenance' chemotherapy. Children on UKALL VIII received the drug daily for the first 27 weeks, and those on UKALL X took it three times weekly throughout treatment.

\section{PRESCRIBING OF 6-MERCAPTOPURINE}

The actual amounts of 6-mercaptopurine prescribed for each child during central nervous system prophylaxis and the first 24 months of 'maintenance' treatment were extracted from the records. They were converted into $\mathrm{mg} / \mathrm{m}^{2}$ for each three month period and then added together. For those diagnosed after 1980 the longest uninterrupted period at the target dose and the number of times that the dose was reduced because of myelosuppression were also extracted.

This was not done for the group diagnosed before 1980 because few children were ever given the target dose and because there was no consistency in the degree of myelosuppression that led to a reduction in dose, so the results could not be compared.

\section{STATISTICAL ANALYSIS}

The Mann-Whitney U test was used to compare medians. Duration of remission between the groups was compared by the log rank test. When considering control of the disease by treatment, deaths during remission and second malignancies were not counted as 'events' but were censored at the time of their occurrence.

\section{Results}

PATIENTS

In the group diagnosed before 1980 there were 30 eligible children (19 boys and 11 girls). Nineteen children were treated according to UKALL V (11 boys and eight girls), and 11 according to UKALL III (eight boys and three girls). The ages at diagnosis ranged from 2 to 11 years (median 5.5), and the diagnostic white cell counts ranged from $0.3-16.3 \times 10^{9} / 1$ (median 4.2). All had 'non-T-cell' acute lymphoblastic leukaemia. At the end of April 1990 length of 
follow up ranged from 27 to 193 months (median 147).

In the group diagnosed after 1980 there were 55 children ( 30 boys and 25 girls). Forty four (24 boys and 20 girls) were consecutively enrolled in UKALL VIII (arms A and B) and 11 (six boys and five girls) were consecutively enrolled in UKALL XA. The ages at diagnosis ranged from 0.3-13 years (median 4) and the white cell counts at diagnosis ranged from $0.8-670 \times 10^{9} / 1$ (median 9.0). The immunological disease types were distributed as follows: $65 \%$ were common acute lymphoblastic leukaemia, $16 \%$ were pre-B, $5 \%$ were null, $5 \%$ were T-cell acute lymphoblastic leukaemia, and 9\% were unclassifiable. At the end of April 1990 the length of follow up ranged from 23 to 121 months (median 76). When this group was matched with the group diagnosed before 1980 , 15 children who had T-cell acute lymphoblastic leukaemia or white cell counts of more than $20 \times 10^{9} / 1$ at diagnosis were excluded to make the groups comparable.

PRESCRIBING OF 6-MERCAPTOPURINE

Group diagnosed before 1980

Before 1980 modifications of the dose of 6mercaptopurine if myelosuppression occurred were left to the clinicians' discretion, with the aim of keeping the white cell count between 2 and $3 \times 10^{9} / 1$. Prescribing of 6-mercaptopurine under these relaxed guidelines was compared with prescribing under the stricter regimens after 1980. Children diagnosed before 1980 received considerably lower cumulative doses of 6-mercaptopurine (range 22106 to $49563 \mathrm{mg}$ ) $\mathrm{m}^{2}$; median 31 103) than comparable patients treated after that date (range 22820 to 50777 $\mathrm{mg} / \mathrm{m}^{2}$; median 40 499) (fig 1). The median difference between the two groups was $7743 \mathrm{mg} /$ $\mathrm{m}^{2}$ (95\% confidence interval (CI) 4141 to 10964 $\mathrm{mg} / \mathrm{m}^{2}, \mathrm{p}<0.0001$ ).

This increase in the amount of 6mercaptopurine that was prescribed cannot be accounted for solely by differences between protocols before and after 1980. The percentage increase in the median prescribed dose after 1980 was $22 \%$, but the average increase in the 'target' cumulative dose of 6-mercaptopurine specified in the protocols after 1980 was only
9\%. The largest difference was in UKALL III 'ordinary modified' $\mathrm{E}$ in which the potential dose of 6-mercaptopurine was $34 \%$ less than the potential dose after 1980 , but only three children in the group diagnosed before 1980 received chemotherapy according to this protocol.

When boys and girls were analysed separately the increase in the amount of 6-mercaptopurine prescribed after 1980 was more pronounced for boys than for girls. Before 1980 boys' doses ranged from 23365 to $49563 \mathrm{mg} / \mathrm{m}^{2}$ (median 31019 ) and after 1980 'standard risk' boys' doses ranged from 29746 to $50777 \mathrm{mg} / \mathrm{m}^{2}$ (median 41711 ). The median difference was $9025 \mathrm{mg} / \mathrm{m}^{2}$ (95\% CI 4264 to $\left.13302, \mathrm{p}<0.004\right)$. For girls doses prescribed before 1980 ranged from 22106 to $39843 \mathrm{mg} / \mathrm{m}^{2}$ (median 31 187) and after 1980 ('standard risk') doses ranged from 22106 to $47175 \mathrm{mg} / \mathrm{m}^{2}$ (median 37560 ). The median difference was $6394 \mathrm{mg} / \mathrm{m}^{2}(95 \%$ CI 540 to $\left.11419 \mathrm{mg} / \mathrm{m}^{2}, \mathrm{p}<0.04\right)$. Among those diagnosed before 1980 there was no significant difference in the amount of 6-mercaptopurine prescribed to boys compared with girls.

\section{Group diagnosed after 1980}

In the group diagnosed after 1980 ('all risk') the median cumulative dose of 6-mercaptopurine for boys was $42099 \mathrm{mg} / \mathrm{m}^{2}$ (range 29746 50777 ) whereas for girls it was $38151 \mathrm{mg} / \mathrm{m}^{2}$ (range $22820-47$ 175) (fig 2). The median difference between the prescribed dose for boys compared with that for girls was $3647 \mathrm{mg} / \mathrm{m}^{2}$ ( $95 \%$ CI 222 to $7468 \mathrm{mg} / \mathrm{m}^{2}, \mathrm{p}<0.03$ ).

The greater tolerance to 6-mercaptopurine shown by boys after 1980 was also reflected in the duration of the longest uninterrupted period they spent at the target dose of $75 \mathrm{mg} / \mathrm{m}^{2}$. Their median time was 19 weeks (range 5-82) whereas for girls it was 13 weeks (range 2-45) $(p<0.05)$ (fig 3). Girls also had more reductions in doses because of myelosuppression that were specified in the protocols (range 5-23, median 14.0) than boys (range $2-20$, median 10.5$)(p<0.03)$.

Non-compliance could potentially have affected these results, but children in this study group were also having red blood cell 6thioguanine nucleotides (an active metabolite of 6-mercaptopurine) measured regularly as part

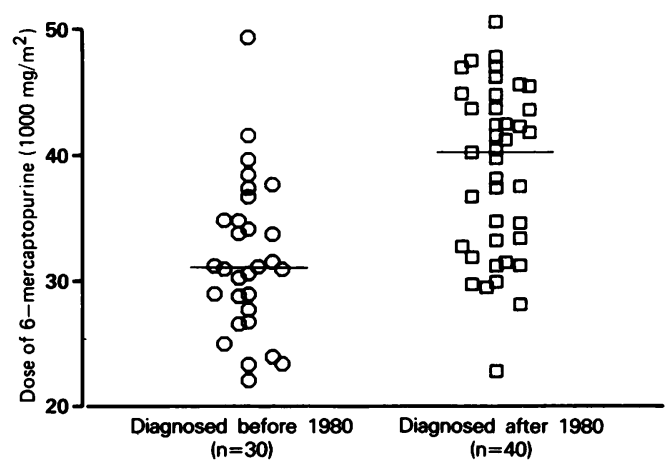

Figure 1 Cumulative dose of 6-mercaptopurine $\left(\mathrm{mg} / \mathrm{m}^{2}\right)$ in study groups before $(n=30)$ and afier $(n=40) 1980$. Horizontal lines indicate median values (before 1980 , $31103 \mathrm{mg} / \mathrm{m}^{2}$, after $\left.1980,40499 \mathrm{mg} / \mathrm{m}^{2}\right)$.

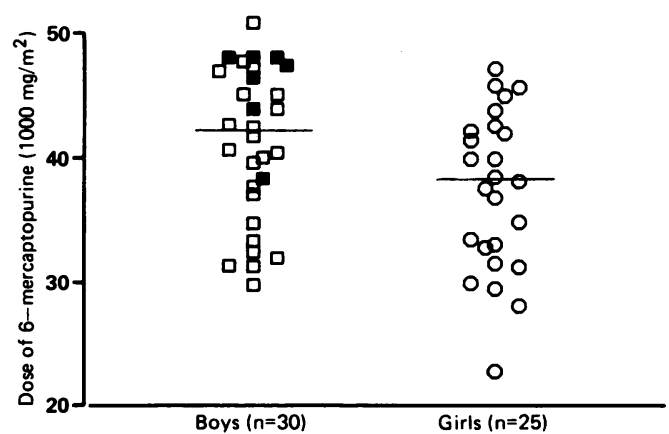

Figure 2 Cumulative dose of 6-mercaptopurine $\left(\mathrm{mg} / \mathrm{m}^{2}\right)$ in boys $(n=30)$ and girls $(n=25)$ diagnosed after 1980 . Horizontal lines indicate median values (boys $42099 \mathrm{mg} / \mathrm{m}^{2}$, girls $\left.38151 \mathrm{mg} / \mathrm{m}^{2}\right)$. Closed symbols indicate patients who subsequently relapsed. 


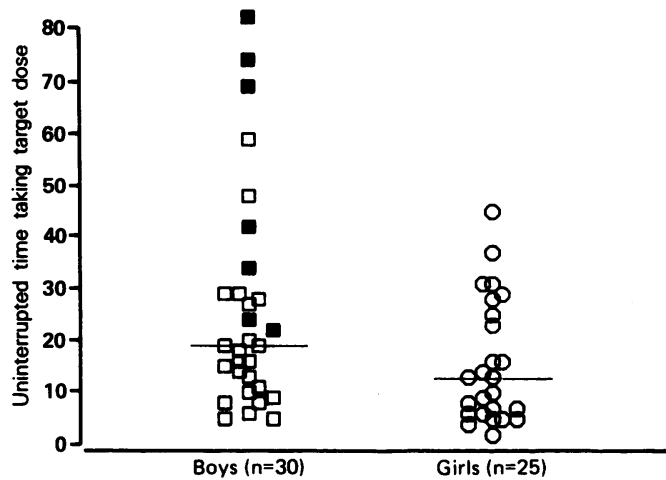

Figure 3 Longest uninterrupted time (weeks) spent at tanget dose of $75 \mathrm{mg} / \mathrm{m}^{2}$ for boys $(n=30)$ and girls $(n=25)$.

Horizontal lines indicate median times (boys 19 weeks, girls 13 weeks). Closed symbols indicate patients who subsequently relapsed.

of another study and non-compliance was not detected by this technique.

\section{OUTCOME OF TREATMENT}

Outcome of treatment among the children studied reflected national trends in the United Kingdom. At the time of analysis there had been nine relapses (two girls and seven boys in the group diagnosed before 1980 compared with four in the group diagnosed after 1980 ('standard risk') who were all boys. Log rank analysis indicated that there was an $18 \%$ improvement after 1980 in those still in first remission at five years $\left(\chi^{2}=3.9, p<0.05 ; 95 \%\right.$ CI 0.08 to $\left.36 \cdot 76 \%\right)$.

In the 'all risk' group diagnosed after 1980, outcome of treatment was also examined in relation to the amount of 6-mercaptopurine that had been given. There were seven relapses in the whole group (all boys). There was an estimated $15 \%$ increase in numbers remaining in remission at five years for those who received less than the group median cumulative dose of 6-mercaptopurine (one relapse) compared with those receiving more than the group median dose (six relapses) $\left(\chi^{2}=3.69, \mathrm{p}<0.07 ; 95 \% \mathrm{CI}\right.$ -3.6 to $33 \cdot 2 \%$ ) (fig 4 ). Five of the seven relapses occurred in the upper quartile for 6mercaptopurine dosage.

There were no differences between the groups above and below the median dose of 6mercaptopurine in diagnostic white cell count or type of leukaemia. There was an apparent

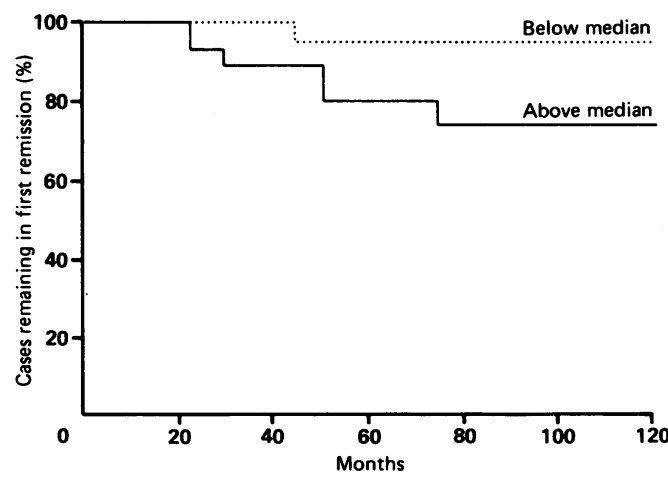

Figure 4 Percentage of cases remaining in first remission for those receiving above the group median dose of $40011 \mathrm{mg} / \mathrm{m}^{2}$ 6 -mercaptopirine $(n=27)$ and those receiving below the median dose $(n=28) ; \chi^{2}=3 \cdot 69, p<0.07$. excess of boys in the group above the median (18 boys and nine girls) and particularly in the upper quartile (11 boys and three girls) but this was not significant.

\section{Discussion}

This single centre study with homogeneity of prescribing practice both before and after 1980 provides objective data to support the suggestion made by the Medical Research Council Working Party that more rigid prescribing criteria for drugs used in 'maintenance' treatment have contributed to the improved outlook for children with acute lymphoblastic leukaemia diagnosed after $1980 .{ }^{1}$ In our patients the amount of 6-mercaptopurine that was prescribed undoubtedly increased after 1980, and much more than could be explained by changes in target doses specified in protocols. This was associated with a significant improvement in relapse free survival.

Because of the parallel adjustment of doses of 6-mercaptopurine and methotrexate during 'maintenance' chemotherapy it also follows that cumulative doses of methotrexate must have increased after 1980 as well. We believe that this is less likely to have had such a large effect on outcome, as the doses of methotrexate that are used are small.

Other studies have also shown an association between the amounts of 6-mercaptopurine and methotrexate prescribed during 'maintenance' chemotherapy and subsequent relapse of leukaemia. ${ }^{6-8}$ In studies in which children were not treated with doses large enough to cause toxicity, ${ }^{67}$ either because of the dose given in the protocol, ${ }^{6}$ or because physicians did not comply, ${ }^{7}$ the children who received less treatment did worse. In contrast, Silberman et al found that a small group of children with acute lymphoblastic leukaemia who received less than half their potential dose of 6-mercaptopurine because of pronounced toxicity related to treatment did better than similar patients who had less toxic reactions.

Our group diagnosed after 1980 show the same paradoxical phenomenon. Within this group most children were being treated with doses large enough to cause toxicity and were occasionally experiencing myelosuppression. Those who had more toxic reactions, and who were therefore being prescribed less 6mercaptopurine seemed less likely to relapse, whereas those who tolerated uninterrupted target doses were more likely to relapse. This observation was not significant, but taken in the context of other studies points to a suggestive trend.

As more children are being prescribed as much 6-mercaptopurine as they can tolerate, individual differences in metabolism of the drug are becoming apparent. In the group diagnosed after 1980 the cumulative dose of 6-mercaptopurine that was tolerated by different patients ranged from $43 \%$ to $97 \%$ of the potential dose using the same prescribing guidelines. This almost certainly reflects underlying differences in the metabolism of 6-mercaptopurine among patients. ${ }^{9}$ 
As well as differences among patients the group diagnosed after 1980 also showed a significant difference in the tolerance of 6mercaptopurine between the sexes, the boys being prescribed more 6-mercaptopurine and spending longer uninterrupted periods at the target dose specified in the protocol than girls using the same prescribing guidelines. This has been noted before over shorter times and presumably reflects a sex difference in the metabolism of 6-mercaptopurine. ${ }^{2}$ This difference in tolerance between the sexes was not noted in the group diagnosed before 1980 . The reason may be that with the earlier 'gentle' approach to treatment, doses of 6-mercaptopurine were not influenced as much by tolerance in either sex. In our study group boys had a worse outcome of treatment than girls, and it was striking that the boys most tolerant of 6-mercaptopurine subsequently relapsed more often.

The worse prognosis for boys with acute lymphoblastic leukaemia remains unexplained but perhaps sex differences in the metabolism of 6-mercaptopurine may in large part be responsible. ${ }^{10}$ It may not be a coincidence that hypoxanthine-guanine phosphoribosyltransferase (the main enzym $\dot{c}$ : responsible for converting the inactive native drug to its cytotoxic metabolites) is sex linked, and we are currently exploring this possibility.

In the meantime the findings of this single centre study clearly indicate that one of the chief differences between treatment protocols before and after 1980 is the amount of 6mercaptopurine that was prescribed during 'maintenance' treatment as a result of changed criteria for reducing doses rather than a difference in recommended maximum doses in the protocols. Improvement in outlook for children in the United Kingdom with acute lymphoblastic leukaemia may in large part be because of this. The future intention to titrate the dose of 6-mercaptopurine to myelotoxicity in all children may prevent children who are more tolerant of the drug being inadvertently undertreated. It may, by the same mechanism, eliminate the difference in prognosis between the sexes.

This work was supported by the Leukaemia Research Fund.

1 Report to the Council by the Working Party on Leukaemia in Childhood. Improvement in treatment for children with acute lymphoblastic leukaemia. The Medical Research Council UKALL Trials 1972-84. Lancet 1986;i:408-11.

2 Lilleyman JS, Lennard L, Rees CA, Morgan G, Maddocks $\mathrm{JL}$. Childhood lymphoblastic leukaemia: sex difference in

3 The Medical Research Council's Working Party on Leukaemia in Childhood. The treatment of acute lymphoblastic leukaemia (ALL) in childhood, UKALL III. The effects of added cytosine arabinoside and/or asparaginase, and a comparison of continuous or discontinuous mercapto purine in regimens for standard risk ALL. Med Pediat Oncol 1982;10:501-10.

4 Report to the Council by the Working Party on Leukaemia in Childhood. Medical Research Council Leukaemia TrialUKALL V: an attempt to reduce the immunosuppressive effects of therapy in childhood acute lymphoblastic leukaemia. $\mathcal{F}$ Clin Oncol 1986;4:1758-64.

5 Peto R, Pike MC, Armitage P, et al. Design and analysis of randomized clinical trials requiring prolonged observation of each patient. II. Analysis and examples. $B r f$ Cancer 1977;35:1-39.

6 Pinkel D, Hernandez K, Borella L, et al. Drug dosage and remission duration in childhood lymphocytic leukaemia. Cancer 1971;27:247-56.

7 Peeters M, Koren G, Jakubovicz D, Zipursky A. Physician compliance and relapse rates of acute lymphoblastic leukaemia in children. Clin Pharmacol Ther 1988;43: leukaemia 228 .

8 Silberman T, Robison LL, Nesbit ME, Sather HN, Ortega JA, Hammond GD. Association between outcome in childhood acute lymphoblastic leukaemia (ALL) and amount of maintenance 6-mercaptopurine (6-MP). Proceedings of the American Society of Clinical Oncology 1985;4:166.

9 Lennard L, Lilleyman JS. Variable mercaptopurine metabolism and treatment outcome in childhood lymphoblastic leukaemia. F Clin Oncol 1989;7:1816-23.

10 Sather M, Miller D, Nesbit M, Heyn R, Hammond D. Difference in prognosis for boys and girls with acute lymphoblastic leukaemia. Lancet 1981;i:739-43. 